\title{
Avaliação das medidas lineares e angulares de cavalos de vaquejada em Araguaína, Tocantins
}

\author{
[Linear and angular measurements in "vaquejada" horses in Araguaina, Tocantins]
}

\section{"Artigo Científico/Scientific Article"}

\author{
Yan Oliveira Lacerda ${ }^{1}$, Fernanda Caroline Rotta Cristino Fioravante ${ }^{1}$, \\ Rajman Schneider Fonseca ${ }^{1}$, Matheus Henrique Dias Rodrigues ${ }^{1}$, Leandro Lopes Nepomuceno ${ }^{2}$, \\ José Américo Soares Garcia ${ }^{3}$, Fabiana Cordeiro Rosa ${ }^{1}$, Jorge Luís Ferreira ${ }^{1}$
}

\author{
${ }^{1}$ Universidade Federal do Tocantins, Campus de Araguaína, Araguaína-TO, Brasil. \\ ${ }^{2}$ Programa de Pós-graduação em Ciência Animal, Faculdade de Veterinária e Zootecnia, Universidade Federal de Goiás, \\ Goiânia-GO, Brasil. \\ ${ }^{3}$ Universidade de Brasília, Campus Darcy Ribeiro, Faculdade de Agronomia e Veterinária, Brasília-DF, Brasil. \\ *Autor para correspondência/Corresponding author: E-mail: jlferreira@uft.edu.br
}

\section{Resumo}

Objetivou-se caracterizar morfologicamente através da metodologia das proporções corporais e angulares para cavalos de vaquejada na região de Araguaína, Tocantins. Foram utilizados 63 animais, de ambos os sexos, sendo mensuradas 17 medidas lineares e 8 angulares, utilizando-se de um hipômetro, fita métrica e um artrogoniômetro. Os resultados observados no estudo, demonstraram uma grande variação na estatura, peso, perímetro torácico, peso vivo, garupa e largura do peito, e houve diferença entre os sexos, principalmente para estatura, peso, perímetro torácico e garupa dos animais, caracterizando-os em animais de impulso, explosão e velocidade, como também de animais de trabalho e de carga (tração). Possivelmente essa diferença possa está associada a maior pressão de seleção das fêmeas, uma vez que a intensidade de seleção nas mesmas é maior que nos machos. Não foi observada uma associação favorável e alta entre medidas dos ângulos dos membros traseiros e dianteiros, o que de certa forma poderia favorecer melhor marcha e andamento, bem como comodidade. Assim, recomenda-se que a seleção de animais seja também direcionada para comodidade e andamento dos animais.

Palavras-chave: Ângulos; esporte; equinos; fenótipo.

\begin{abstract}
The objective of this study was to characterize the body and angle proportions of "vaquejada" horses in the Araguaína region, Tocantins. Sixty-three animals of both sexes were used to linear and angular measurements in "vaquejada" horses in Araguaína, Tocantins measure 17 linear and angular measurements, using a hypometer, tape measure, and an arthrogoniometer. The results observed in the study showed a large variation in height, weight, thoracic perimeter, weight, croup, and breast width. There was a difference between the sexes, mainly for height, weight, thoracic perimeter, and croup of the animals, characterizing them in animals of impulse, explosion, and speed, as well as of work and of traction. Possibly this difference may be associated with higher selection pressure of females since the selection intensity in females is higher than in males. There was no favorable and high association between the rear and front limb angle measurements, which in a way could favor better gait, as well as comfort. Therefore, it is recommended that the selection of animals is also directed to the comfort and gait of the animals.
\end{abstract}

Keywords: Angles; sport; horses; phenotype.

\section{Introdução}

O Brasil possui maior rebanho de equinos da (ANUALPEC, 2017), e a indústria equina mobiliza América Latina, com 5,8 milhões de cabeças ao ano R\$ 16 bilhões de reais (BRASIL, 2016). 
Segundo dados das organizações que controlam as raças equinas no Brasil, apenas 700.000 animais são registrados (ABQM, 2017), e aproximadamente 596 mil estão distribuídas na região Norte do país, sendo que 274.297 mil localizadas no Estado do Tocantins, e em torno de 31 mil na região de Araguaína, e 4,5 mil no município de Araguaína (IBGE, 2017).

Nesta região, assim como no Nordeste brasileiro, além do uso na lida com o gado, os equinos são muito utilizados em competições equestres, onde destaca-se a vaquejada. Com a evolução da vaquejada houve a necessidade da utilização de animais que fossem mais adequados, devido principalmente às exigências a sua funcionalidade. Fonseca et al. (2016) trabalhando com cavalos de tração na região de Araguaína, verificaram que muitos animais foram provenientes de descarte de fazendas, e de acasalamentos entre animais nativos e equinos da raça Quarto de Milha.

Nas provas de vaquejada, os animais são extremamente exigidos, com esforço físico de alta intensidade, mas de curta duração, o que requer uma largada rápida, bruscas mudanças de direção e paradas e alta força física para derrubada do boi (Mota e Corrêa, 2004; Meneses et al., 2014; Costa et al., 2016)

De acordo com Ramos et al. (2014), independentemente da raça, a seleção de equinos para seu trabalho obedece a padrões zoométricos, em harmonia com sua funcionalidade. A avaliação morfométrica é importante e muito utilizada para a escolha de equinos, especialmente para atividades esportivas, de carga e tração. Essas avaliações também podem ser utilizadas no estudo do crescimento desses animais e irão proporcionar a definição das taxas de crescimento e aptidões específicas de cada raça (Fonseca et al., 2016).

$\mathrm{O}$ estudo da morfometria em equinos proporciona avaliação das medidas corporais em conjunto, aumentando a capacidade de entender às mudanças ocorridas com o tempo, e possibilitando desenvolvimento de critérios de seleção e objetivos (Zamborlini et al., 1996; Costa et al., 2016).

Todavia, apesar da relevância do assunto, estudos sobre a conformação corporal e o equilíbrio de equinos competidores de modalidades esportivas são raramente encontrados na literatura (Donofre et al., 2014). Dessa forma, este trabalho teve por objetivo utilizar a metodologia das proporções corporais e angulares para avaliação morfológica e de equilíbrio de cavalos participantes da modalidade de vaquejada, na região de Araguaína, Tocantins.

\section{Material e Métodos}

A pesquisa foi conduzida no período de setembro de 2015 a março de 2016, analisando equinos (machos e fêmeas) da raça Quarto de Milha, e animais mestiços, utilizados em provas de vaquejada realizadas na região de Araguaína, Tocantins. $\mathrm{O}$ experimento foi conduzido considerando todas as exigências de bem-estar animal e das resoluções do comitê de ética em pesquisa animal.

Foram realizadas mensurações de características morfométricas, angulares e determinação de índices de conformação em 63 equinos pertencentes a haras conhecidos e idôneos no município, que detinham animais participantes de provas de vaquejada. Do total de animais (63) participantes da prova esportiva, 47 eram machos $(74,6 \%)$ e 16 fêmeas $(25,4 \%)$. A definição dos haras deu-se pela sua localização na região, e assim houve a seleção apenas de animais que eram participantes de provas de vaquejada.

As mensurações foram realizadas com os animais posicionados em piso plano, com os membros torácicos e pélvicos alinhados sob estação forçada, utilizando-se como ferramentas o hipômetro para as medidas de altura, fita métrica para os perímetros, e um artrogoniômetro para as medidas angulares.

Foram mensuradas medidas lineares de Perímetro Torácico (PT); Altura de Cernelha (AC); Altura da Garupa (AG); Perímetro da Canela (PC); Largura de Peito (LP); Comprimento da cabeça (Ccab); Comprimento do pescoço (Cpesc.); Comprimento do dorso-lombo (CDL); Comprimento da garupa (CGar); Comprimento da espádua (Cesp.); Largura da cabeça (Lcab); Largura da garupa (LGar); Altura codilho ao solo (ACS); Altura do joelho (AJ); Perímetro do joelho (PJ); Altura de jarrete (AJA); e Perímetro de antebraço $(\mathrm{PAB})$. Os ângulos mensurados foram: escápulo-umeral (AEU), úmero-radial (AUR), metacarpo-falangiano (AMF), coxo-femural (ACF), fêmur-tibial (AFT), tíbio-metatarsiano (ATM) e metatarso-falangiano (AMF), descritos conforme Santiago et al. (2013).

O delineamento experimental foi inteiramente casualizado, e todas as variáveis (medidas lineares e angulares) foram submetidas a análise de variância pelo modelo estatístico: 


$$
y_{i j}=\mu+S_{i}+e_{i j} \text {,em que, } y_{i j} \text { é }
$$

a variável dependente (medidas lineares e angulares), $S_{i}$ é o efeito fixo do sexo (macho ou fêmea); e $e_{i j}$ é o erro aleatório associado a cada observação, normalmente distribuído $e \sim N\left(0, \sigma_{e}^{2}\right)$.

As médias foram comparadas pelo teste de Tukey a 5\% de significância, por meio do procedimento GLM, enquanto as estimativas de correlação de Pearson calculadas por meio do procedimento CORR. Todas as análises foram realizadas por meio do pacote estatístico Statistical Analyses System (SAS, 2002).

\section{Resultados}

Na Tabela 1 está sumarizado a estatística descritiva das medidas lineares para cavalos utilizados em provas de vaquejada na região de Araguaína, Tocantins. Os equinos, em geral, apresentaram porte mediano (eumétricos), com média de peso de $453,96 \pm 41,22 \mathrm{~kg}$, altura de cernelha 1,49 $\pm 0,05 \mathrm{~m}$, e 75\% (47/63) dos animais estudados apresentaram AC de 1,45 a 1,52 m.

Tabela 1. Estatísticas descritivas de medidas lineares de cavalos da raça Quarto de Milha utilizados em provas de vaquejada na região norte do Estado do Tocantins.

\begin{tabular}{cccccc}
\hline & Média & DP & CV & MIN & MAX \\
\hline PT (m) & 1,78 & 0,05 & 3,00 & 1,70 & 1,71 \\
AC (m) & 1,49 & 0,05 & 3,05 & 1,40 & 1,59 \\
AG (m) & 1,51 & 0,04 & 2,77 & 0,18 & 1,62 \\
PC(m) & 0,20 & 0,01 & 4,45 & 0,37 & 0,22 \\
LP (m) & 0,45 & 0,03 & 6,82 & 0,54 & 0,51 \\
Ccab (m) & 0,59 & 0,02 & 2,72 & 0,64 & 0,93 \\
Cpesc (m) & 0,78 & 0,06 & 7,93 & 0,69 & 0,88 \\
CDL (m) & 0,78 & 0,05 & 5,96 & 0,55 & 0,71 \\
Cgar (m) & 0,65 & 0,04 & 5,93 & 0,55 & 0,68 \\
Cesp (m) & 0,61 & 0,03 & 5,28 & 0,19 & 0,23 \\
Lcab (m) & 0,21 & 0,01 & 4,56 & 0,49 & 0,58 \\
Lgar (m) & 0,53 & 0,02 & 3,68 & 0,77 & 0,94 \\
ACS (m) & 0,84 & 0,04 & 4,48 & 0,39 & 0,50 \\
AJ (m) & 0,44 & 0,02 & 5,28 & 0,30 & 0,34 \\
PJ (m) & 0,32 & 0,01 & 3,33 & 0,43 & 0,59 \\
AJA (m) & 0,54 & 0,03 & 5,05 & 393,04 & 0,49 \\
PAB (m) & 0,42 & 0,03 & 7,61 & 5,08 & 5,43 \\
PESO (Kg) & 453,96 & 41,22 & (m)
\end{tabular}

$\mathrm{PT}=$ Perímetro torácico; $\mathrm{AC}=$ altura da cernelha; $\mathrm{AG}=$ altura da garupa; $\mathrm{PC}=$ perímetro da canela; $\mathrm{LP}=\mathrm{Largura}$ do peito; $\mathrm{Ccab}=$ Comprimento da cabeça; Cpesc $=$ Comprimento do pescoço; $\mathrm{CDL}=$ comprimento dorso-lombo; $\mathrm{Cgar}=\mathrm{Comprimento}$ da garupa; Cesp = Comprimento da espádua; Lcab = Largura da cabeça; Lgar = Largura da garupa; $\mathrm{ACS}=$ Altura do codilho ao solo; $\mathrm{AJ}=$ Altura do joelho; $\mathrm{PJ}=$ Perímetro do joelho; $\mathrm{AJA}=$ Altura do jarrete; $\mathrm{PAB}=$ Perímetro do antebraço; $\mathrm{PESO}=$ Peso vivo verdadeiro; $\mathrm{DP}=$ Desvio-padrão; $\mathrm{CV}=$ Coeficiente de variação; $\mathrm{MIN}=$ Mínimo; $\mathrm{MAX}=$ Máximo.

Fonte: Informações dos autores.

Foi verificada diferença significativa $(\mathrm{p}<0,05)$ entre os sexos para as medidas lineares de perímetro torácico (PT), largura da garupa (Lgar) e Peso vivo (PV), tendo as fêmeas apresentados valores superiores aos machos. As médias de AC (altura da cernelha), para machos e fêmeas, foram de $1,52 \mathrm{~m}$ e $1,54 \mathrm{~m}$, respectivamente.

Considerando o cavalo Quarto de Milha, como exemplar de animal competidor na região, o padrão racial sugerido é de um animal com estatura maior nos machos que nas fêmeas, apresentando uma variação média de $1,52 \mathrm{~m}$ a $1,62 \mathrm{~m}$, na estatura (ABQM, 2017).

As medidas de PT variaram de $1,77 \mathrm{~m} \mathrm{e}$ $1,81 \mathrm{~m}$, em machos e fêmeas, respectivamente, verificando-se maior proporção nas fêmeas. A média do peso vivo das fêmeas foi $7,15 \%$ superior à média dos machos, com média de 480,61 kg \pm 41,22. Essa característica é altamente correlacionada $(0,86)$ com o perímetro torácico.

A determinação do peso corporal dos animais é importante para avaliar o crescimento e o estado nutricional, estabelecer o valor de venda do animal e ajustar o arraçoamento (Lucena et al., 2015).

Em relação aos índices verificou-se que para o índice dáctilo-torácico (IDT), os animais foram considerados de sela e estão em uma posição intermediária entre animais hipermétricos e eumétricos, justificando mais uma vez que possivelmente a composição genética dos animais necessita de ajustes, uma vez que, para os demais 
índices (IC, IG1, IG2, ICom1 e ICom2) classificaram os animais do estudo como animais de tração.

$\mathrm{Na}$ Tabela 2 estão apresentadas as estatísticas descritivas das medidas angulares de cavalos da raça Quarto de Milha utilizados em provas de vaquejada. Verificou-se no presente estudo que as maiores variações encontradas foram para os ângulos escapulo-umeral $(\mathrm{CV}=10,88 \%) \mathrm{e}$ ângulo coxo-femural $(\mathrm{CV}=10,66 \%)$.

Tabela 2. Estatísticas descritivas de medidas angulares de cavalos da raça Quarto de Milha utilizados em provas de vaquejada na região norte do Estado do Tocantins.

\begin{tabular}{cccccc}
\hline & Média & DP & CV & MIN & MAX \\
\hline AEU & $72,86^{\circ}$ & $7,71^{\circ}$ & 10,58 & $60^{\circ}$ & $80^{\circ}$ \\
AUR & $126,67^{\circ}$ & $8,42^{\circ}$ & 6,65 & $110^{\circ}$ & $150^{\circ}$ \\
AMF & $150,32^{\circ}$ & $4,74^{\circ}$ & 3,15 & $140^{\circ}$ & $170^{\circ}$ \\
ACF & $66,19^{\circ}$ & $7,05^{\circ}$ & 10,66 & $60^{\circ}$ & $80^{\circ}$ \\
AFT & $78,41^{\circ}$ & $7,23^{\circ}$ & 9,22 & $70^{\circ}$ & $110^{\circ}$ \\
ATM & $148,89^{\circ}$ & $8,05^{\circ}$ & 5,41 & $140^{\circ}$ & $160^{\circ}$ \\
AMF & $159,84^{\circ}$ & $6,35^{\circ}$ & 3,97 & $150^{\circ}$ & $170^{\circ}$ \\
\hline
\end{tabular}

AEU = Ângulo escapulo-umeral; AUM = Ângulo úmero-radial; AMF = ângulo metacarpo-falangeano; ACF = ângulo coxofemural; AFT = ângulo fêmur-tibial; ATM = ângulo tíbio-metatarsiano; AMF = ângulo metatarso-falangeano DP = Desvio-padrão; CV = Coeficiente de variação; MIN = Mínimo; MAX = Máximo.

Fonte: Informações dos autores.

Essa amplitude, para ambos ângulos, denota satisfatoriamente a grande diferença de composição genética dos animais utilizados em provas de vaquejada da região. Uma vez que os ângulos com maior amplitude estão relacionados com capacidade de impulsão, flexibilidade, redução de atritos verticais, explosão em arrancadas e maior propulsão dos membros posteriores (Menezes et al. 2014; Rezende et al. 2014; Costa et al. 2016).

As correlações entre os comprimentos (Ccab, Cpesc, CDL, Cgar, Cesp) foram em média moderadas (r: 0,30$)$. Os maiores valores $(0,86)$ foram observados entre AC e AG. Entre as larguras (Lcab, Lgar, LP), verificou-se maior correlação entre LP e Lgar $(0,56)$, sendo, em geral, com os ângulos considerados de baixa magnitude $(0,21)$.

De maneira geral, as correlações entre comprimentos, alturas, perímetros e larguras foram classificadas como moderada $(0,79)$, sendo altamente significativas $(\mathrm{P}<0,001)$ entre as medidas de PT com Cesp e AG com AC, e também entre PC e CDL, LP e Lgar, Cpesc e AC, e CDL e $\mathrm{AC}$.

As Tabelas 3 e 4 representam as correlações e significâncias entre as medidas lineares e angulares. Para os ângulos a maior correlação se deu entre os ângulos ACF e ATM $(0,52)$, sendo altamente significativo, entretanto, essa correlação é de magnitude intermediária. Relacionando ângulos anteriores e posteriores não foi observado uma correlação significativa e de magnitude alta, demonstrando a necessidade de uma harmonia maior entre os ângulos anteriores e posteriores, o que promoveria um melhor desempenho nas provas de vaquejada.

Tabela 3. Valores de correlação e significância entre as medidas angulares de cavalos da raça Quarto de Milha criados na região de Araguaína, Tocantins.

\begin{tabular}{lccccccc}
\hline & AEU & AUM & AMF & ACF & AFT & ATM & AMF \\
\hline AEU & 1 & $0.24^{*}$ & 0.23 & -0.12 & $0.28^{*}$ & -0.10 & 0.04 \\
AUM & & 1 & $0.26^{*}$ & 0.08 & -0.008 & 0.13 & 0.08 \\
AMF & & & 1 & $0.32^{* *}$ & -0.12 & -0.03 & 0.05 \\
ACF & & & 1 & $-0.31^{*}$ & $0.52^{* * *}$ & 0.05 \\
AFT & & & & 1 & -0.11 & 0.06 \\
ATM & & & & & 1 & $0.43^{* *}$ \\
AMF & & & & & & 1 \\
\hline
\end{tabular}

AEU = Ângulo escapulo-umeral; AUM = Ângulo úmero-radial; AMF = ângulo metacarpo-falangeano; ACF = ângulo coxofemural; $\mathrm{AFT}=$ ângulo fêmur-tibial; $\mathrm{ATM}=$ ângulo tíbio-metatarsiano; $\mathrm{AMF}=$ ângulo metatarso-falangeano. $*=(\mathrm{P}<0,05) ; * *=(\mathrm{P}<0,01)$; $* * *=(\mathrm{P}<0,0001)$.

Fonte: Informações dos autores. 
Tabela 4. Valores de correlação e significância entre as medidas lineares de cavalos da raça Quarto de Milha criados na região de Araguaína, Tocantins.

\begin{tabular}{|c|c|c|c|c|c|c|c|c|c|c|c|c|c|c|c|c|}
\hline & $\mathbf{A C}$ & AG & PC & LP & Ccab & Cpesc & CDL & Cgar & Cesp & Lcab & Lgar & ACS & $\mathbf{A J}$ & PJ & AJA & PAB \\
\hline PT & $0.40^{*}$ & $0.34^{*}$ & $0.49^{* * *}$ & $0.41 * *$ & 0.24 & 0.29 & $0.35^{*}$ & $0.26^{*}$ & $0.61^{* * * *}$ & 0.21 & $0.59 * * *$ & 0.16 & 0.14 & $0.44^{* *}$ & -0.01 & $0.38^{*}$ \\
\hline AC & & $0.86^{* * * *}$ & $0.52^{* * *}$ & 0.23 & $0.47 * * *$ & $0.61 * * *$ & $0.56^{* * *}$ & $0.52^{* * * *}$ & $0.34 *$ & 0.23 & 0.20 & $0.39 *$ & $0.40^{*}$ & $0.54 * * *$ & $0.38^{*}$ & 0.20 \\
\hline AG & & & $0.43^{*}$ & $0.30^{*}$ & $0.40^{*}$ & $0.42^{*}$ & $0.42 *$ & $0.39 *$ & $0.32 *$ & 0.18 & 0.15 & $0.47^{* * * *}$ & $0.45^{* *}$ & $0.42 * *$ & $0.44 * *$ & 0.18 \\
\hline PC & & & & $0.28 *$ & $0.36^{*}$ & $0.32 *$ & $0.51 * * *$ & $0.47 * * *$ & $0.27 * *$ & -0.005 & $0.25^{*}$ & 0.18 & 0.15 & $0.45^{* *}$ & $-0.10^{*}$ & 0.33 \\
\hline LP & & & & & 0.01 & -0.04 & $0.36^{*}$ & 0.23 & 0.18 & 0.10 & $0.56^{* * * *}$ & $0.38 *$ & 0.09 & 0.16 & 0.10 & $0.42 * *$ \\
\hline Ccab & & & & & & 0.11 & 0.23 & $0.30^{*}$ & -0.01 & 0.19 & 0.12 & 0.14 & 0.24 & 0.17 & -0.005 & -0.10 \\
\hline Cpesc & & & & & & & $0.57 * * *$ & $0.35^{*}$ & $0.44 * *$ & 0.23 & 0.03 & 0.04 & $0.28^{*}$ & $0.55^{* * *}$ & 0.18 & 0.16 \\
\hline CDL & & & & & & & & $0.25^{*}$ & 0.23 & -0.02 & $0.45^{* *}$ & $0.37 *$ & $0.42^{* *}$ & $0.37 *$ & 0.16 & $0.39 *$ \\
\hline Cgar & & & & & & & & & 0.69 & $0.31^{*}$ & 0.10 & 0.03 & -0.10 & $0.47^{* * *}$ & 0.06 & $0.25^{*}$ \\
\hline Cesp & & & & & & & & & & 0.21 & $0.31^{*}$ & 0.12 & 0.23 & $0.40^{*}$ & 0.20 & $0.29^{*}$ \\
\hline Lcab & & & & & & & & & & & -0.02 & -0.13 & -0.00 & 0.17 & 0.15 & -0.04 \\
\hline Lgar & & & & & & & & & & & & $0.42 * *$ & 0.16 & 0.20 & 0.05 & $0.47^{* * *}$ \\
\hline ACS & & & & & & & & & & & & & $0.47 * * *$ & 0.19 & $0.24 *$ & $0.38^{*}$ \\
\hline AJ & & & & & & & & & & & & & & 0.17 & $0.50^{* * *}$ & 0.11 \\
\hline PJ & & & & & & & & & & & & & & & -0.03 & $0.49^{* * *}$ \\
\hline AJA & & & & & & & & & & & & & & & & -0.02 \\
\hline
\end{tabular}

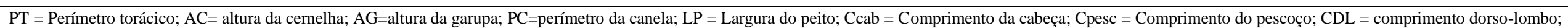

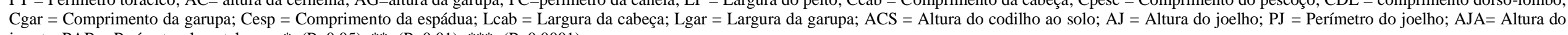
jarrete; $\mathrm{PAB}=$ Perímetro do antebraço; $*=(\mathrm{P}<0,05) ; * *=(\mathrm{P}<0,01) ; * * *=(\mathrm{P}<0,0001)$

Fonte: Informações dos autores. 


\section{Discussão}

Os animais utilizados no presente estudo não pertencem a uma raça específica, embora a grande maioria são descendentes de cruzamentos de animais da raça Quarto de Milha. Nessa raça, o registro de medidas lineares e angulares não é realizado de forma obrigatória, para efeito de registro de indivíduos junto à associação de criadores, justificando assim, a necessidade do presente estudo.

Ademais, embora o número de animais utilizados para obtenção de um perfil possa ser considerado pequeno, o volume de dados aqui levantados é expressivo, visto o tamanho reduzido de haras e animais destinados às provas de vaquejada na região, e a falta de trabalhos que venham demonstrar o perfil desses animais, bem como, apresentar estratégias para uma correta seleção dos animais para o objetivo-fim.

Os resultados observados no estudo, demonstraram uma grande variação na estatura, peso, perímetro torácico, peso vivo, garupa e largura do peito. Segundo Meira (2013), animais de corrida apresentam estatura mais elevada, maior peso e comprimento, já animais de trabalho possuem estatura mais compacta e menor comprimento corporal, sendo uma possível explicação para a formação desses animais, uma vez que o porte foi mediano, e houve diferença entre os sexos, principalmente para estatura, peso, perímetro torácico e garupa dos animais, caracterizando-os em animais de impulso, explosão e velocidade, como também de animais de trabalho e de carga (tração).

Outra possibilidade pode estar também relacionada a formação do plantel na região, pois os animais que disputam o esporte possivelmente são resultantes de cruzamentos de animais de linhagem de corrida e trabalho, uma vez que as medidas morfológicas variam entre o observado nas linhagens. A morfologia dos equinos apresenta relação funcional com o desempenho dos animais em suas diversas atividades, como corrida, freio, corrida de tambor, entre outras (Meira et al., 2013).

A largura da garupa é uma medida extremamente importante do ponto de vista prático, pois está associada à movimentação e força dos membros posteriores. Assim, a seleção de animais com maior velocidade reflete em cavalos mais compridos e com garupas mais desenvolvidas proporcionando animais com melhor desempenho em corridas (Pereira et al., 2014).
Segundo Jones (1987), as proporções do peito, tanto na profundidade quanto na largura, são extremamente importantes, pois, conferem resistência ao equino. Comparando com outros estudos (Miserani et al., 2002; Santiago et al., 2013), em outras raças (mangalarga marchador e pantaneiro, respectivamente), os resultados aqui apresentados demonstram que esses animais possuem medidas mais adequadas ao exercício da vaquejada, uma vez que tal esporte, exige animais com maior necessidade de oxigenação em detrimento do maior esforço físico praticado nas provas de vaquejada, uma vez, que um mesmo animal, em sua grande maioria, é submetido a mais de uma competição por dia.

As medidas corporais mais mencionadas na literatura para predizer o peso são o perímetro torácico, o comprimento corporal, a altura da cernelha e da garupa e o comprimento da garupa. Embora existam discrepâncias sobre qual medida individual deve ser utilizada para predizer o peso, a acurácia da predição tem sido geralmente alta especialmente quando mais de uma medida for considerada (Heirinchs et al., 1992; Khalil e Vaccaro, 2002). Possivelmente essa diferença possa está associada a maior pressão de seleção das fêmeas, uma vez que a intensidade de seleção nas mesmas é maior que nos machos. Embora não existem evidencias cientificas sobre esse fato, é comum observar, nos criatórios da região, que éguas, selecionadas apenas em quesitos fenotípicos e de desempenho principalmente em vaquejadas, sejam matrizes em cruzamentos com garanhões da raça Quarto de Milha.

Com relação aos machos, os pesos encontrados nesta pesquisa são inferiores aos reportados por Pimentel et al. (2012), Ramos et al. (2014) e Costa et al. (2016). Entretanto, Costa et al. (2016) não encontraram efeito do dimorfismo sexual para esta medida. A média de peso vivo encontrada em ambos os sexos, foi inferior a maioria dos resultados observados em outras pesquisas (Pimentel et al., 2012; Ramos et al., 2014; Costa et al., 2016).

Em animais que praticam atividades esportivas sempre se requer força, impulsão e velocidade. Verificou-se que os animais do estudo sofreram maior pressão de seleção para força, necessitando priorizar velocidade, principalmente em virtude de medidas angulares e outras medidas lineares, como perímetro da canela, comprimento 
da espádua, altura do codilho ao solo, largura da cabeça, largura do peito e massa corporal.

Para as medidas de angulação os resultados obtidos neste trabalho foram semelhantes àqueles encontrados por Costa et al. (2016), que ao avaliarem equídeos das raças Brasileira de Hipismo, Bretão Postier e Jumento Brasileiro relataram valores de $60,79^{\circ}$ pra $\mathrm{AEU}, 122,25^{\circ}$ para AUR, $145,42^{\circ}$ para AMF, $85,25^{\circ}$ para ACF, $146,67^{\circ}$ para ATM e $153,96^{\circ}$ para AMF.

Observou-se que para a medida angular escapulo-umeral (AEU), os resultados foram abaixo dos apresentados por Meneses et al. (2014) e Costa et al. (2016) para animais de velocidade e força. Segundo Meneses et al. (2014) o ângulo escapulo-umeral é importante para o impulso, pois escápulas com maior inclinação apresentam maior área para inserção muscular e possibilitam maior flexibilidade e amplitude dos movimentos dos membros torácicos. Assim, um animal com maior angulação escapulo-umeral estaria associado com maior capacidade de impulsão, flexibilidade articular e concomitantemente maior passada e redução de atritos verticais.

O comprimento da passada está relacionado com o comprimento dos membros e com o ponto de rotação dos mesmos, o que invariavelmente estão relacionados com as medidas morfométricas e angulares (Stashak, 1994), pois os membros anteriores suportam de 60 a $65 \%$ do peso do corpo. Uma espádua bem inclinada permite ao membro anterior realizar movimentos mais bruscos $\mathrm{e}$ avantes. Por outro lado, uma espádua mais vertical restringe a amplitude desse movimento e, consequentemente, diminui o comprimento da passada (Harris, 1993).

Portanto, considerando-se as medidas dos ângulos dos membros anteriores e o comprimento da cabeça, pescoço e altura da cernelha pode-se sugerir que nos animais avaliados, há uma necessidade de uma pressão de seleção mais eficiente para cavalos de explosão e velocidade, uma vez que as medidas (métricas e angulares) foram inferiores a outros estudos.

Nos cavalos da raça Quarto de Milha é recomendável que a coxa seja mais inclinada, o que proporciona maior propulsão nos membros posteriores e, consequentemente, maior explosão para arrancada na corrida (Meneses et al., 2014). Os valores médios encontrados nesta pesquisa são inferiores aos reportados por Nascimento (1999), Lage et al. (2009), Meneses et al. (2014), Santiago et al. (2014) e Costa et al. (2016).
A medida angular fêmur-tibial (AFT) foi superior à reportada por Costa et al. (2016) e inferior à reportada por Meneses et al. (2014). Segundo Meneses et al. (2014), quanto menor a medida desse ângulo, melhor a flexão dos jarretes, o que facilita a maior propulsão dos membros pélvicos e melhor flexibilidade para as paradas brusca dos animais Quarto de Milha.

O ACF foi menor aos descritos na literatura (Santiago et al., 2013; Meneses et al., 2014; Costa et al., 2016), sendo superior aos reportados por Rezende et al. (2014). Segundo Camargo e Chieffi (1971) e Nascimento (1999), garupas mais obliquas ou inclinadas tendem a proporcionar coxas mais musculosas, com maior desenvolvimento dos músculos glúteos e ísquiostibiais. Assim, uma coxa menos inclinada está associada a uma garupa mais horizontal, menor desenvolvimento glúteo e menor propulsão.

Não foi observada uma associação favorável e alta entre medidas dos ângulos dos membros traseiros e dianteiros, o que de certa forma poderia favorecer melhor marcha e andamento, bem como comodidade. Assim, recomenda-se que a seleção de animais seja também direcionada para comodidade e andamento dos animais, pois a associação alta e significativa entre os ângulos dos membros dianteiros e traseiros poderia ser benéfica para propulsão e paradas bruscas.

Segundo Ribeiro (1988), nem todos os ângulos têm a mesma ação e efeito. Os de abertura anterior possuem forte ação nos movimentos do animal e, por isso, são chamados de impulsores, enquanto os de abertura posterior são denominados complementares ou de ligação. Dessa forma, para haver harmonia e equilíbrio nos andamentos, é necessário que estes ângulos, dos membros anteriores e posteriores, mantenham perfeita concordância nas respectivas aberturas e direções. Os membros anteriores estão relacionados à comodidade do andamento e os posteriores, ao rendimento do andamento.

A função principal dos locomotores dianteiros é suportar o peso, absorver choques e levantar o animal do solo durante o seu deslocamento, enquanto os locomotores posteriores proporcionam a impulsão principal. Portanto, o locomotor posterior é projetado para empurrar o corpo para frente, paralelo ao solo, ao passo que o locomotor dianteiro tem a função dupla de empurrar e alçar (Toledo, 1985). 


\section{Conclusão}

Existe variabilidade fenotípica de equinos de vaquejada em relação às medidas lineares e angulares, cujas regiões anatômicas apresentaramse associadas com a função desempenhada pelo animal. Entretanto, no plantel em estudo há uma necessidade de ações de seleção, principalmente relacionadas ao acasalamento dirigido que venha favorecer o desenvolvimento de uma linhagem mais alinhada ao perfil esportivo que a vaquejada exige.

\section{Conflito de Interesse}

Os autores declaram não existir conflito de interesse.

\section{Referências}

ANUALPEC: Anuário da agricultura brasileira. São Paulo: FNP, 2017. Disponível em: <http:// anualpec.com.br>. Acesso em: 07 nov. 2017.

Associação Brasileira de Criadores de Quarto de Milha - ABQM. Disponível em $<$ http://abqm.com.br/conteudos/quarto-demilha/quarto-de-milha-no-brasil >. Acesso em 20 jan. 2017.

BRASIL. Ministério da Agricultura, Pecuária e Abastecimento. 2016. Revisão do estudo do complexo do agronegócio cavalo. Disponível em: $<$ http://www.agricultura.gov.br/assuntos/cam aras-setoriais-

tematicas/documentos/camarassetoriais/equideocultura/anosanteriores/revisao-do-estudo-do-complexodo-agronegocio-do-cavalo/view $>$. Acesso em: 20 set. 2017.

Camargo, M.X.; Chieffi, A. In:__. Ezoognósia: revisão atualizada da obra-exterior dos grandes animais domésticos. São Paulo: SA/CPA/Instituto de Zootecnia, 1971.

Costa, M.D.; Mendes, L.J.; Maruch, S.; Ramirez, P.A.; Meneses, A.C.A.; Martins Neto, T.; Ruas, J.R.M.; Chamone, J.M.A. Efeito da composição genética nas carcaterísticas de conformação em equinos. Arquivo Brasileiro de Medicina Vetereinária e Zootecnia, 68(6): 1629-37, 2016.

Donofre, A.C.; Puoli Filho, J.N.P.; Ferreira, I.E.D.P.; Mota, M.D.S.D.; Neto, C. Equilíbrio de cavalos da raça Quarto de Milha participantes da modalidade de três tambores por meio de proporções corporais. Ciência Rural, 44(2): 327-32, 2014.
Fonseca, R.S.; Schmidt, A.B.; Guilherme, G.O.; Silva, M.P.B.; Lacerda, Y.O. Padrão morfométrico de equinos de tração no município de Araguaína, Tocantins. Revista Acadêmica Ciência Animal, 14: 195-202, 2016.

Harris, M.; Raviv, A. Differences of opinion make a horse race. The Review of Financial Studies, 6(3): 473-506, 1993.

Heinrichs, A.J.; Roogers, G.W.; Cooper, J.B. Predicting body weight and wither height in Holstein heifers using body measurements. Journal of Dairy Science, 75: 3576-3581, 1992.

Ibge. Censo demográfico 2010. Disponível em: < http://censo2010.ibge.gov.br >. Acesso em: 10 jul. 2017.

Jones W. In:_. Reprodução eqüina: genética e criação de cavalos. São Paulo: Roca, 1987. Cap. 16:417-39.

Khalil, R.; Vaccaro, L.B. weights and measurements in dual purpose cows: their interrelation and association with genetic merit for three production traits. Zootecnia Tropical, 20: 11-30, 2002.

Lage, M.; Bergmann, J.; Procópio, A.; Pereira, J.; Biondini, J. Associação entre medidas lineares e angulares de equinos da raça Mangalarga Marchador. Arquivo Brasileiro de Medicina Vetereinária e Zootecnia, 61(4): 968-79, 2009.

Lucena, J.E.C; Vianna, S.A.B.; Berbari Neto, F.; Sales Filho, R.L.M.; Diniz, W.J.S. Estudo comparativo das proporções morfométricas entre garanhões. Semina: Ciências Agrárias, v. 36(1) 353-366, 2015.

Meira, C.T.; Fortes, M. R. S.; Farah, M. M.; Portoneto, 1. R.; Curi, R. A.; Moore, S. S. A genome-wide association study for height at withers in racing quarter horse. Association for the Advancement of Animal Breeding and Genetics, 20:420-423, 2013.

Meneses, A.C.A.; Costa, M.D.; Maruch, S.; Moreira, P.R.; Neto, T.M. Medidas lineares e angulares de animais da raça Quarto de Milha utilizados em uma prova de vaquejada. Revista Brasileira de Ciência Veterinária, 21(4)256-261, 2014.

Mota, M.; Corrêa, M. Parâmetros genéticos para índice de velocidade em cavalos da raça Quarto de Milha. Archive Zootecnia, 53:38790, 2004. 
Nascimento, J. In:_. Mangalarga marchador: tratado morfofuncional. Belo Horizonte: Abccmangalarga Marchador. 1999.

Pereira, G.L.; Meira, C.T.; Silva, J.A.I.; Chardulo, L.A.L.; Curi, R.A. Estimativas de parâmetros genéticos para características morfométricas em cavalos quarto de milha de corrida. Ciência \& Tecnologia Fatec-JB, 6(esp):448, 2014.

Pimentel, M.L.; Camara, F.V.; Dantas, R.A.; Freitas, Y.B.; Dias, R.V.; Souza, M.V. Biometria de equinos de vaquejada no Rio Grande do Norte, Brasil. Acta Veterinaria Brasilica, 5(4):376-9, 2012.

Ramos, T.N.M.; Cardoso, D.; Oliveira, J.V.; de Miranda Bomfim, C.A. Características zoométricas de equinos de raças definidas e não definidas criadas na região de Araçatuba, SP. Boletim de Indústria Animal, 71(3):23440, 2014.

Rezende, M.P.G.; Souza, J.C.; Mota, M.F.; Jardim, R.J.D.; Ramires, G.G.; Silva, R.M.; Souza, C.F. Morfometria corporal de equinos utilizados em trabalho, esporte e lazer em três municípios do Mato Grosso do Sul. Veterinária e Zootecnia, 21(4):569-83, 2014.

Ribeiro, D.B. In:_. O cavalo: raças, qualidades e defeitos. Rio de Janeiro: Globo, 1988.
Santiago, J.M.; de Rezende, A.S.C.; Fonseca, M.G.; Abrantes, R.G.P.; Lage, J.; Lana, Â.M.Q. Comparação entre as medidas morfométricas do rebanho atual de machos Mangalarga Marchador e dos campeões da raça. Boletim de Indústria Animal, 70(1):46-52, 2013.

Santiago, J.M.; de Rezende, A.S.C.; Lana, Â.M.Q.; Fonseca, M.G.; Abrantes, R.G.P.; Lage, J.; Andrade, J.M.; Resende, T.M. Medidas morfométricas do rebanho atual de fêmeas Mangalarga Marchador e das campeãs da raça. Revista Brasileira de Saúde e Produção Animal, 15(1):141-148, 2014.

Stashak, T. S. In:_. Claudicação em eqüinos segundo Adams. 4a ed. São Paulo: Roca, 1994. 1112p.

Statistical analysis system user's guide. Carey, NC, SAS Institute Inc. 9 $9^{\text {a }}$ version, 2002.

Toledo, A.P. In:_. Mecânica de sustentação e locomoção dos eqüinos. 177p. São Paulo: Parma, 1985.

Zamborlini, L.C.; Bergmann, J.A.G.; Pereira, C.S.; Fonseca, C.G.; Carneiro, A.S. Estudo genético-quantitativo de medidas lineares de eqüinos da raça Mangalarga Marchador-I. Estimativas dos fatores de ambiente e parâmetros genéticos. Revista Brasileira de Ciência Veterinária, 3(2):33-37, 1996. 